\title{
Image Extraction of ECG Signals on Human Emotions Using Wavelet Transformation
}

\author{
Jaenal Arifin ${ }^{1}$, Norma Amalia ${ }^{2}$, Yulian Zetta Maulana ${ }^{3}$ \\ \{jaetoga@ittelkom-pwt.ac.id, norma@ittelkom-pwt.ac.id, yulian@ittelkom-pwt.ac.id\} \\ Study Program S1 Teknik Elektro, Institut Teknologi Telkom Purwokerto (ITTP), Jl. D.IPanjaitan \\ No.128, Purwokerto ${ }^{13}$, Program Studi S1 Teknik Telekomunikasi, Institut Teknologi Telkom Purwokerto \\ (ITTP), Jl. D.IPanjaitan No.128, Purwokerto ${ }^{2}$
}

\begin{abstract}
Data processing of human heart's signals can be learned and applied in several studies, whether in psychological subject or in monitoring the condition of the human heart. A human's emotional aspect could be observed when the person recorded his heartbeat activities. The state of emotion observed in a normal, happy and sad conditions. The first stage of this research consists of measuring, recording, printing out the ECG data and scanning. The second stage, which named as pregeneration of the ECG image data stage, consists of median filtering and determining the threshold value and morphology. The third stage uses thinning and spatial-to-time methods for processing its ECG image.The last stage is named as characteristic extraction which uses a wavelet transform. From the characteristic extraction, the average of energy signal is obtained.
\end{abstract}

Keywords: electrocardiography (ECG), wavelet transformation and physiological signal processing.

\section{Introduction}

Electrocardiography (ECG) is a recording of heart's electrical activity. Heart electrical activity is generated from the process of depolarizing from cell to cell and spreading throughout the heart. This activity produces an electric current flow in the body that can be detected by electrodes on the skin's surface [1] [2]. The human body is a good conductor, so that the impulse formed by the heart can be spread throughout the body. Generally, the bioelectric potential emitted by the heart can be measured by electrodes placed in various positions on the surface of the body. Graphs recorded in this recording are called electrocardiography. Electrocardiographic signals have a specific shape that can be used as a reference to determine the condition of the human heart. ECG signals are recorded using an electrocardiograph device. An ECG signal has a shape that is shown in Figure 1 


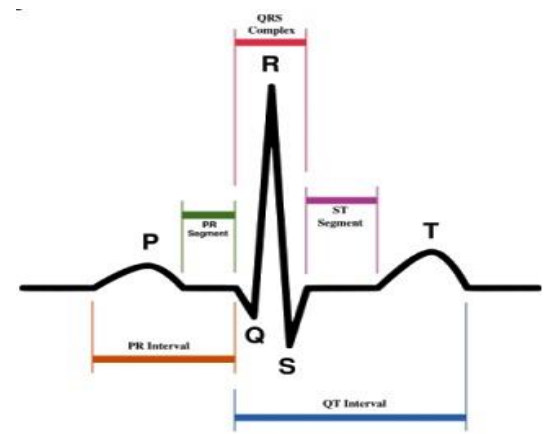

Fig. 1. ECG Wave [3].

The $\mathrm{P}$ wave shows the atrial depolarization triggered by the SA node, the PR segment indicates the stopping impulse at the AV node. The QRS wave indicating ventricular depolarization. The ST segment shows the condition of no impulse caused by the refractory period in the myocardial cell and the T wave shows repolarization [4]. In this research, wavelet transformation method is used, because the wavelet transform method can be used to analyze (observe) non stationary signals. The advantages of the wavelet transform is that the signal characteristics can be learned locally with details result, according to the scale. This property is very useful because it has a transient component with a short life time and has different characteristics on different scales or has a singularity. The use of wavelet transforms is based on the fact that with wavelet transforms we can get much better time and frequency resolution than other methods such as fourier transform and short time fourier transform. In addition, data analysis in the time and frequency domain is important and must be conducted to study the behavior of non stationary signals. Besides, it can also be used to analyze data on the time and amplitude domain and also on the frequency and power domain (spectrum). The wavelet transformation is expected to help detecting human emotions that can be extracted from the average energy generated by ECG signals. In biomedic, signals that is produced is usually a nonstationary signal [5]. In a non-stationary signal, the frequencies change all the times.In order to know the shape of the signal and the duration that separates between these two signals, the methods must be conduct more thoroughly. The ECG signal abnormality can be determined from the reduced or excessive duration of time and the normal signal frequency determined by the cardiovascular expert. The objectives of this research are to perform measurements using electrocardiography (ECG), identify human heart signals and recognize emotions. Analyzing the average signal results of electrocardiographic signals in order to identify a person's emotions in a normal, happy and sad condition.

\section{Literature Study}

The heartbeat signal system can be studied and applied in several psychological studies [6] and can also be applied for monitoring the condition of humant's heart cond [7] [8]. Joshua Proulx et al [9], research is about the development and evaluation of the Bluetooth electrocardiogram sensor which is designed to transmit medical data to mobile phones and the 
data can also be displayed and stored on mobile phones. The purpose of the research is that sensors can detect patient's heart and monitor them from remote locations in real-time. Ratih [1] in her research suggests that the built system can monitor the health of the human heart by using a wireless sensor network connected to a certain interface to a PC (personal computer) so that it can function like an ECG (Electrocardigraphy). In his research, Stefan [10], the heartbeat can give clues to the personality traits of a person. Kim [11] mentioned in his research that psychological emotional changes in listening to music can be conducted by placing 4 (four) biosensors named Electromyogram (EMG), Electrocardiogram (ECG), Respiration (RSP) and Skin Conductivity (SC). The classification of observed musical emotions are positive music with high passion, negative music with high passion, positive music with low arousal and negative music with low arousal. For the Electrocardiogram (ECG), the features which are used are an Interbeal Interbeat (R-R) and heart rate variability (HRV). Bhojwani [12] in his research suggests that the developed method can approach and reproduce physiological signals in simulators that is programmed using wavelet filtering. This method used template designing techniques from existing data sources, which then became the basic of biomedical signal made by this simulator. Physiological signal simulated on electrocardiogram, blood pressure, breathing, derivative of chest impedance $(\mathrm{dz} / \mathrm{dt})$, and photopletheysmogram. Templates are also designed for premature ventricular contraction conditions, left ventricular vibration and bundle branch block in electrocardiogram. The software is designed in MATLAB, and DATAQ Instruments DI-720. The data acquisition system used can display the simulated output. Evaluation of this simulator model is conducted in both quantitative and qualitative terms. The results prove that using wavelets for reconstruction of physiological signals minimizes distortion and maintains the dominant feature in any simulated signal. Measurement of heart electrical activity performed by Rizal [13] has designed wireless LAN Electrocardiography. In this design, the electrocardiographic devices are portable and can be connected wirelessly. The purpose of this device is to monitor ECG signals. Here's a schematic of the ECG wireless LAN system as shown in Figure 2.

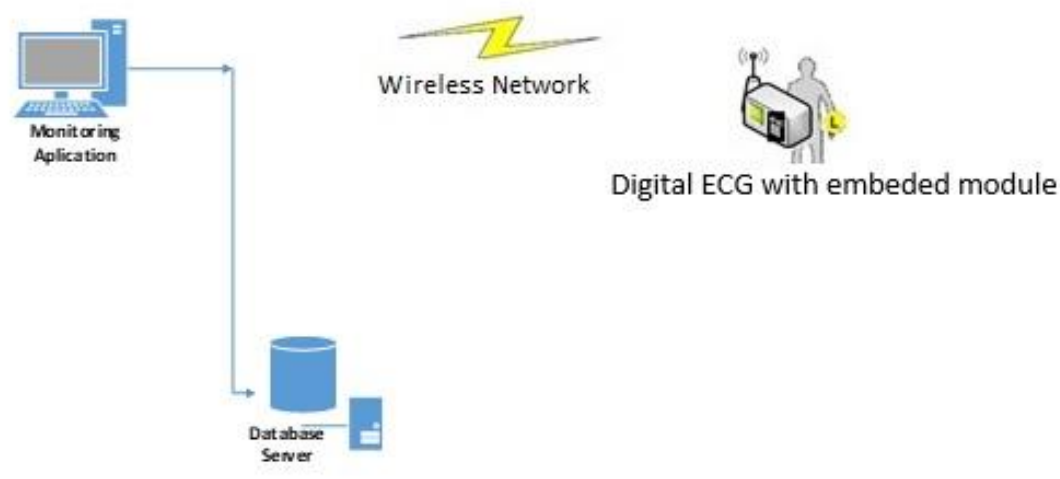

Fig. 2. Wireless System.

The patient's ECG signal is acquired by using a single channel ECG device then the data is sent via wireless LAN. The data is received by the server and recorded and then analyzed. The following hardware sections of the single channel ECG can be seen as in figure 3 . 


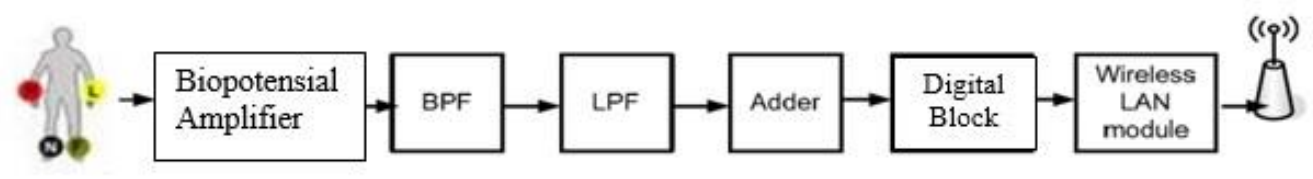

Fig. 3. Diagram Block for Transmitter.

The ECG signal is acquired using electrodes then amplified using biopotensial and filtered using BPF to remove noise at low frequency. Next filtering is conducted by using LPF and the level is increased by using adder to remove negative part of ECG signal, so the signal can be accepted by ADC. The conclusion of this device is that the signal conditioning section has amplified the ECG signal to a suficient level to transmit and that the receiver has been able to receive the transmitted ECG signal. The measured transmission distance can be up to 70 meters with an average delay of $<20 \mathrm{~ms}$. Some of the problems that exist are unstable signal acquisition devices where the movement of the user or humans can cause significant noise.So we need another research to design and create adaptive filters that can eliminate the effect of user movement. The next problem is the newly created wireless LAN network in the form of AdHoc network point to point.This causes the transmission of new ECG data can be conducted by one computer from one transmitter device.

Agrafioti [14] et al suggests that there is a solution based on empirical mode decomposition to dynamically detect emotions on ECG signals. Its classification feature is based on instantaneous frequency (Hilbert-Huang transform) and local oscillations in each mode. The observed parameters use active passion and passive passion. In his research Chendi Wang, Feng Wang proposed an emotional analysis method based on heart rate variability [15]. His research proposed a method for evaluating human emotions and stress based on heart rate variability (HRV). First, the experimental scheme has been designed to induce four types of emotions and to observe the change in response at the electrocardiogram that has been set in the laboratory. Second, the denoising method based on wavelet transformation can be proposed to process a cloudy ECG signal, findings are obtained by various physiological features of various domain analyzes, including time, frequency. Nonlinear analysis can be proposed to find the best relevant emotional features and relate them to emotional states. Jing et al. [16] pointed out that the introduction of emotions based on physiological signals is an important field of research. This study was first performed with conditions of joy and sadness. Electrocardiogram signals are obtained from 391 volunteers through stimulation from sensors that have been attached to certain parts. Determination of P, QRS, T waves is performed using discrete wavelet transform (DWT), which is useful for extracting ECG features through computer media. Using the Tabu Algorithm (TS), the best combination of emotional features is selected for classification. Findings of KNN (k-nearest neighbor) algorithm triggers can be proposed in the study and used to classify test data. This study demonstrates the feasibility of the method searched for ecwectective features. And it is practical to implement TS and KNN (k-nearest neighbor) classifier triggers for emotional recognition based on ECG signals.

Lutfi et al. [17] suggested that his system was able to analyze and clarify the abnormalities of ECG signals using Continuous Wavelet Transform (CWT) and Artificial Neural Network (ANN). ANN configuration used 20,000 neurons in the input layer, 50 neurons in the hidden layer, and 3 neurons in the output layer. ECG signals used are taken from the MIT-BIH 
Arrhythmia database, including normal pulse signals and bundle branch block pulse signals (BBB). The results of this CWT computation show significant time-frequency characteristic difference between normal signal and BBB signal. In testing the ANN classification performance with previously trained data (25 data for 3 classes: normal, left bundle branch block, right bundle branch block), each class is $100 \%$ recognizable. Tests with untrained data, ANN recognizes data with an accuracy of $87.04 \%$. In cross-validation testing, ANN showed good performance with $87 \%$ sensitivity and $95 \%$ specificity. The results of these tests demonstrate the effectiveness of the introduction of ECG signal patterns using CWT and ANN.Chebil et al. [18] suggest that the conversion of ECG data from the manual into digital form is very important. The process of digitizing ECG data includes selecting ECG, extracting trace ECG, unwanted data filtering, applying pixel scale and output integrating the ecg waveform. ECG data already in digital form can be easily stored, accessed or analyzed for the purposes of medical diagnosis or research. In his research proposed some improvement of the existing digitalization process by choosing the appropriate image resolution before scanning. The relationship between pixels, voltage values (mv) and time (time) can be created automatically. The study focuses on determining the minimum PPI value to reduce the computational load required during the digitizing process.

\section{Research Methods}

Here is the methodology used in this study. The design phase of the system is divided into several stages such as measurement and retrieval of ECG data from subject or volunteer, data collection, scanning stage, pre-processing data (changing image to grayscale, median filter, threshold determination, and morphology), thinning method, transformation spatial to time and at the final stage of self extraction.

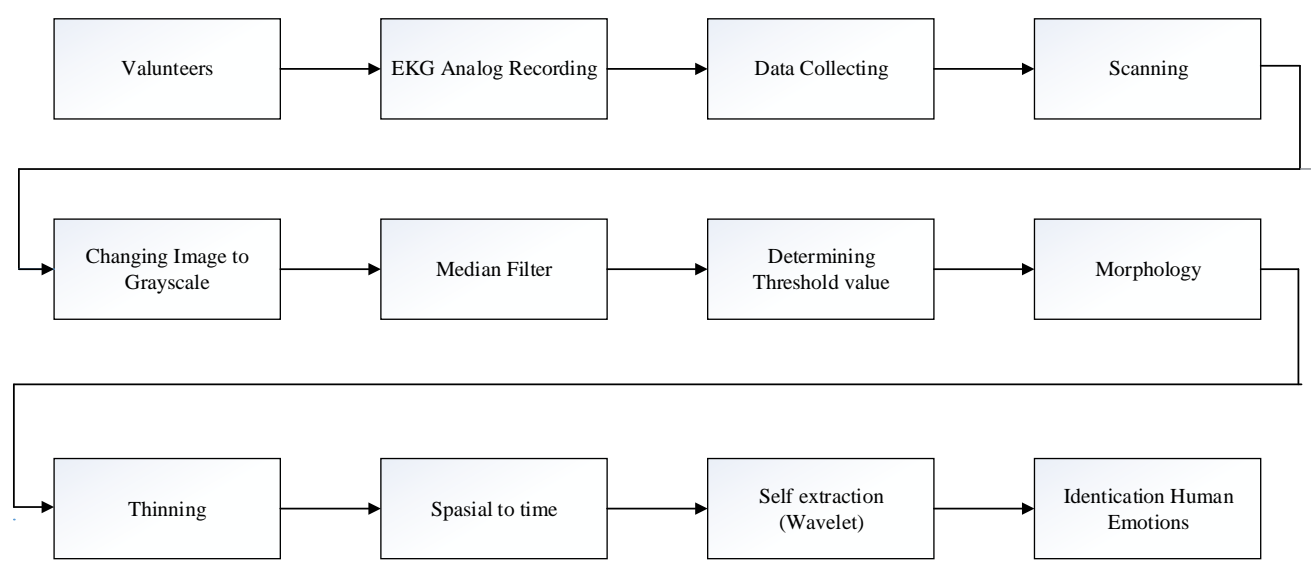

Fig. 4. Design of ECG image signal processing system.

In this study, we conduct electrocardiographic measurements, recording, and data collection with 6 channels and 12 channels using ECG 2000 bionet brands. After ECG signal 
data is obtained then it is processed using supporting software. From analog image converted to digital image, then we conduct pre-processing data, method of thinning, spatial transformation to time and finally self extraction using wavelet sysmlet. Recording of ECG signalsis performed by using 6 channels (I, II, III, aVR, aVL and aVF) with different treatments of human conditions. The condition is feeling happy and feeling sad. Low Pass Filter (LPF) that is used in this ECG device (ECG 2000) is $150 \mathrm{~Hz}$. The purpose of this filter is to remove signals of frequencies which is greater than $150 \mathrm{~Hz}$ [19] [2]. The following flow chart measurement system with ECG 2000 (6 channel) is shown in Figure 5

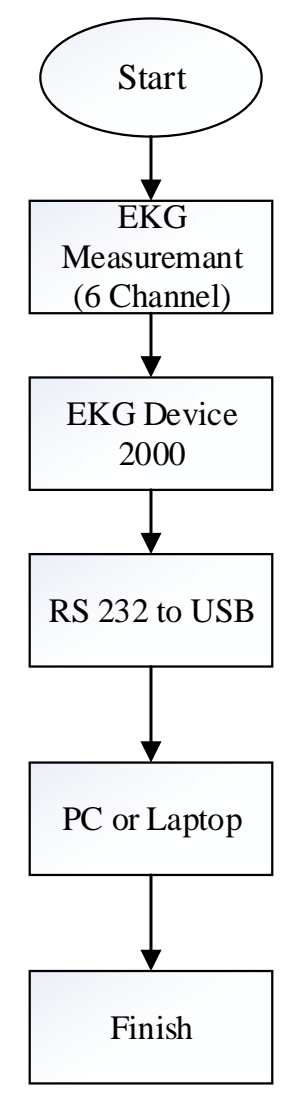

Fig. 5. Measurement Using ECG 2000. 
In the measurement process, some disturbances can occur, such as interference from electrical signals (AC), the volunteers movement when the volunteers breathe in the middle of measurement process and the contraction of muscles and internal organs of volunteers during the measurement. The interference can be solved by activating each filter based on the type of interference. For example for solving interference on electrical signals (AC), we can use filter which is set in $50 \mathrm{~Hz}$ (the electricity frequency that is used in Indonesia). The disturbance caused by volunteer's movement during breathing can be solved by activating base line drift filter. The disturbances that is caused by muscle contractions and internal organs of the subject or relative can be solved by activating the EMG filter [20] [19]. Here is the position of the electrodes positioning at when measuring using ECG2000, which is shown in Figure 6.
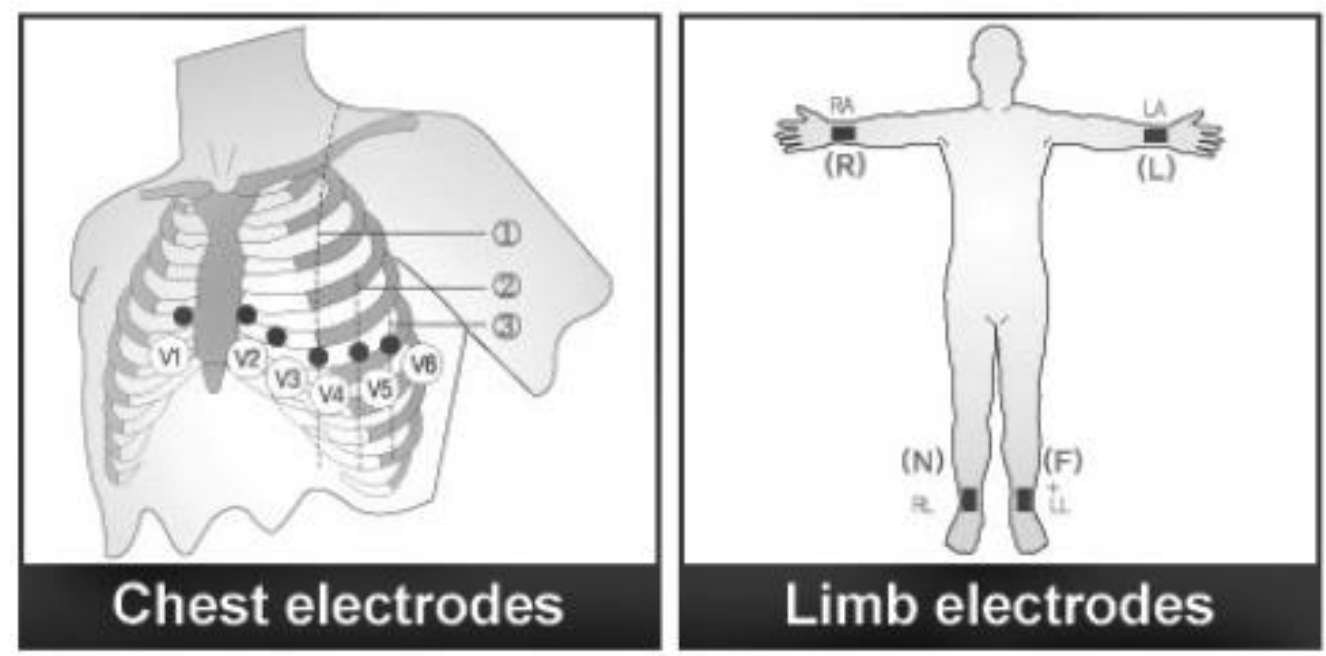

Fig. 6. Electrode Positioning in measurement process.

Caption 6 is as follows:

$\mathrm{N}(\mathrm{RL})=$ right foot

$\mathrm{F}(\mathrm{LL})=$ left foot

$\mathrm{R}(\mathrm{RA})=$ right hand

$\mathrm{L}(\mathrm{LA})=$ left hand

For measurement or recording using 12 channels, the positioning of electrodes are located at points I, II, III, aVR, aV1, aVF, V1, V2, V3, V4, V5 and V6. While the measurement or recording by using only 6 channels, the positioning of electrodes are located at point I, II, III, $\mathrm{aVR}, \mathrm{aVl}$ and aVF. When the electrodes are being installed, every device should be checked, such as checking the connectivity system before making ECG measurements, checking whether there is no mechanical hazard that can put the volunteers in danger. checking the condition of the cable or accessories which is attached to the volunteers. Finally, we must make sure that all the measuring functions in the unit is working properly. The subject or volunteers must be in a comfortable conditions, so that we can give ECG gel in the position where electrode will be attached. The electrode positioning is shown in figure 6. 


\section{Result and Discussion}

Here are examples of ecg signal images from measurements that is conducted. The data is still an ecg signal image. Results of ECG image recording in file.tif format

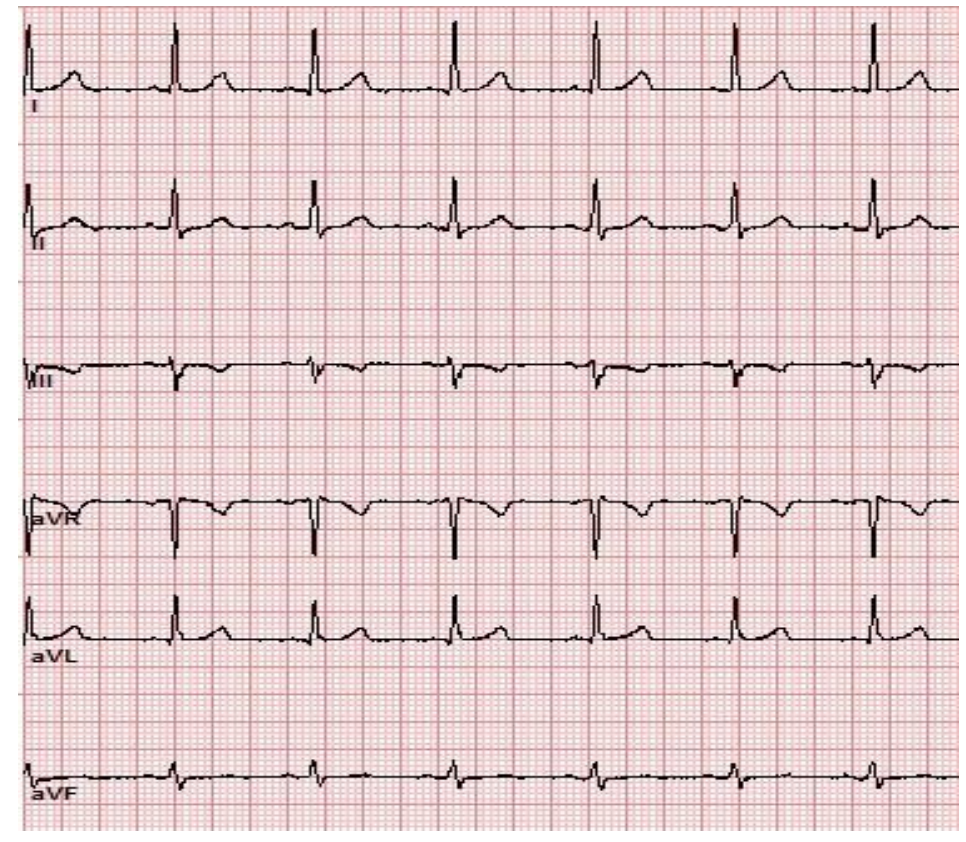

Fig. 7. examples of ecg signal images from measurement results.

There are 6 waves of ECG signal from The Data that we get from measurement. Each data is representing every condition. In this research for the sad condition is created by providing stimulus from listening to music, reading the story or watching the movie. After that, we can observed the average energy of ECG signal on each channel.From measurement data which is in the form of print out, then we conduct scanning process. This scanning process is still in the form of data in a spatial area and still has a grid, but it is in the form of digital data. Here are the stages of pre-processing image processing for ecg signals.

1. Real image ECG signal

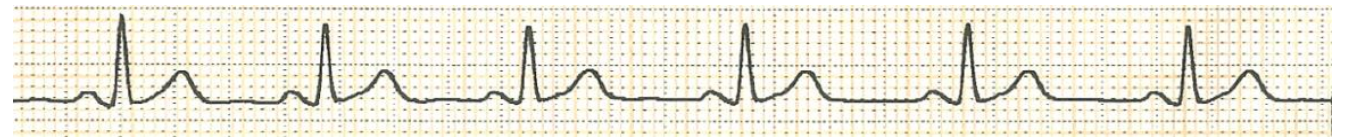

Fig. 8. Real Image ECG signals.

2. Changing image ECG to grayscale 


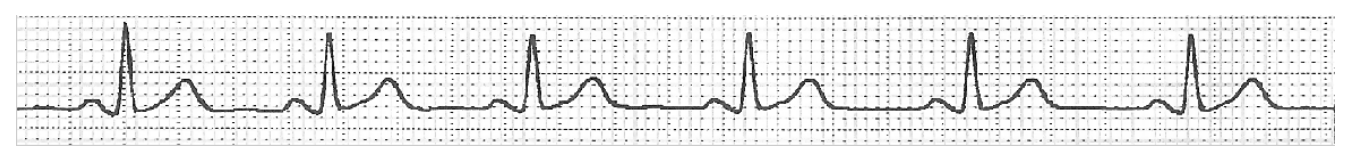

Fig. 9. Changing image ECG to grayscale.

3. Using a Median Filter ( $\operatorname{size} 3$ x 3)

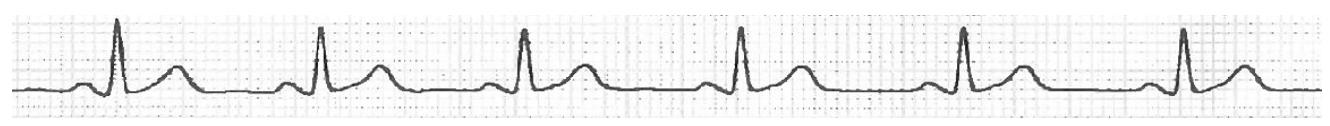

Fig. 10. Image of ECG signal using Median Filter.

4. Determining Threshold value

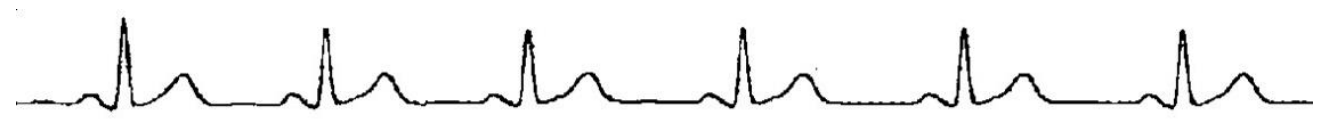

Fig. 11. Image of ECG Signal Threshold Value.

5. Morphology Dillation

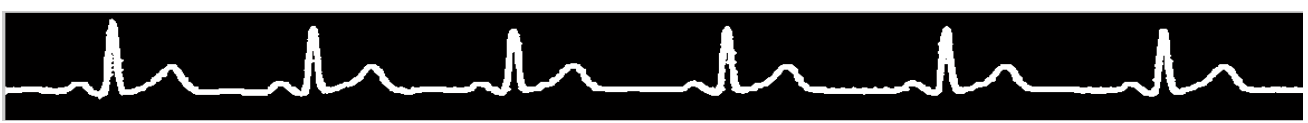

Fig. 12. Image of ECG Signal with Morphology Dillation.

6. ECG Image result with morphology Dillation and Complemet

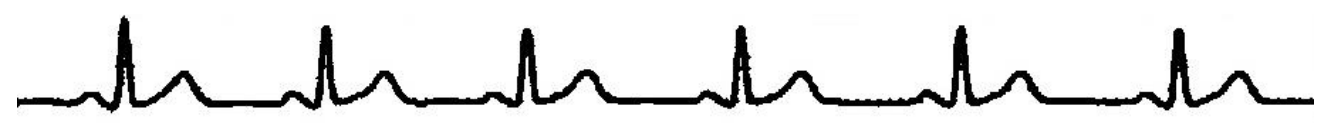

Fig. 13. Results of pre-processing the image of the ECG signal.

In the pre-processing stage the original ecg signal image is converted to grayscale. From the image of the grayscale ECG signal is filtered, using the median filter. This median filter has

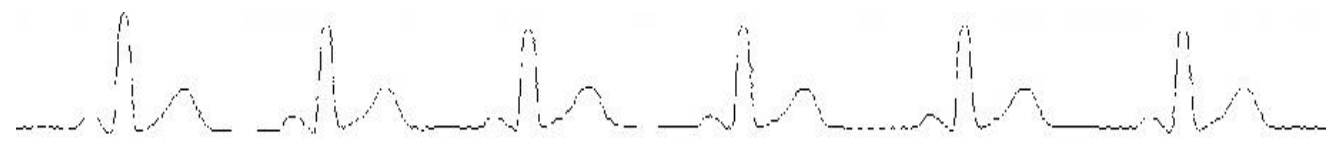


the purpose of removing noise. Noise that exists in the ECG signal image is salt and paper. After the median filter is complete, then the threshold value is determined. The final stage in the preprocessing of the ecg signal is using a dilation morphology. In the processing of this ecg image it reduces a region that is thick or clumped into units with single pixel, so that the ECG image is transformed into pixel lines. Here is the image after the thinning process.

Fig. 14. Result of ECG image after going through the thinning method.

The framework representation of an image from the thinning process has many advantages, such as the relatively small size (due to large amounts of data reduction) and can keep the important pattern. These components can be used when analyzing the shape, one of which is the image of this ECG.Validation of measurements in spatial to time created to determine the accuracy of data after processed by the software. The original measurement data is the print out of the ECG image measurement result. Furthermore the data is processed with the software. The following is a comparison of the original ECG measurement data and ECG measurement data after processing with software

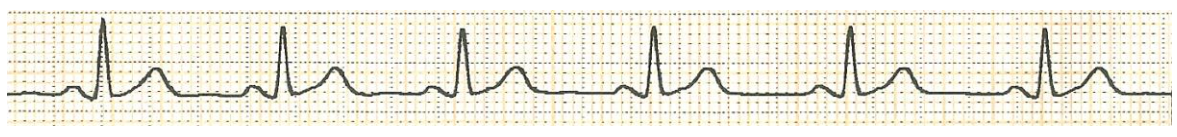

Fig. 15. Real data of ECG measurement.

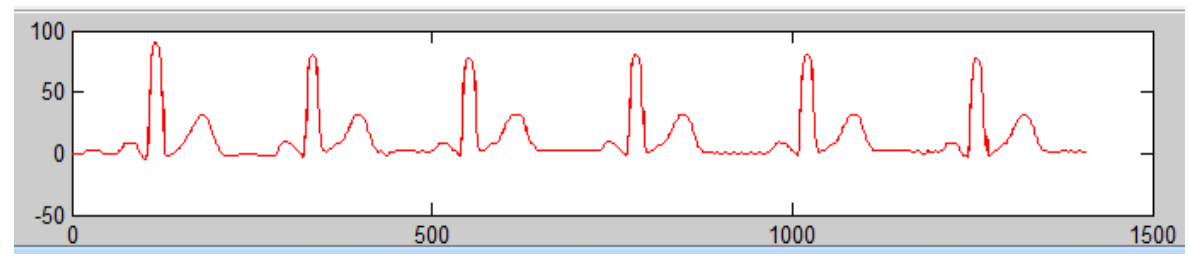

Fig. 16. The simulated data of ecg Signal processed by software.

From the two images (figure 15 and figure 16) it can be seen that there are 6 waves of ecg signals. The comparison results in Figures 15 and 16 are to maintain the information contained in the ECG signal. Figure 16 shows the presence of Cartesian axes (x and y). The y axis shows the amplitude (pixel) and the $\mathrm{x}$ axis shows the signal length (pixel). From the validation of the ecg measurement results, it shows that the validation is correct, while maintaining the information contained in the ecg signal. The following is a comparison of the results of the ECG validation signals

Table 1. Comparison ECG signal validation Result

\begin{tabular}{llll}
\hline $\begin{array}{l}\text { Observed } \\
\text { Parameter }\end{array}$ & $\begin{array}{l}\text { Real ECG Signal } \\
\text { (a) }\end{array}$ & $\begin{array}{l}\text { Simulation ECG Signal } \\
(\mathrm{b})\end{array}$ & $\begin{array}{l}\text { Error } \\
(\mathrm{a}-\mathrm{b}) / \mathrm{a} * 100 \%\end{array}$ \\
\hline P Wave & $0,008 \mathrm{~S}$ & $0,075 \mathrm{~S}$ & $6,25 \%$ \\
QRS Wave & $0,16 \mathrm{~S}$ & $0,15 \mathrm{~S}$ & $6,25 \%$ \\
T wave & $0,22 \mathrm{~S}$ & $0,21 \mathrm{~S}$ & $4,5 \%$
\end{tabular}




\begin{tabular}{llll} 
Peak/ & $0,75 \mathrm{mV}$ & $0,77 \mathrm{~S}$ & $2,67 \%$ \\
R-R Interval & $0,74 \mathrm{~S}$ & $0,73 \mathrm{~S}$ & \\
\hline
\end{tabular}

From the table resulting from the ECG signal validation comparison, the average error is $4.2 \%$. This value is taken from the average of each parameter observed. This parameter is a supporting component of the ECG signal. From the results of the comparison, it shows that the validation is good. Extraction of ECG signal features using discrete wavelet transforms. Feature extraction is done by decomposing the ecg signal. ECG signal measurements performed time and day vary, according to the willingness and condition of the subject or volunteer. The following are the results of the extraction of ecg features on happy, normal and sad conditions

Table 2. Average Normalization Energy for ECG Signal in a happy condition.

\begin{tabular}{llrrrrrr}
\hline Observed Parameter & A10 & D10 & D9 & D8 & D7 & D6 \\
\hline Average Energy & 0,7173 & 0,0082 & 0,0108 & 0,2021 & 0,1317 & 0,0307 \\
Normalizing Average Energy & 1 & 0,0039 & 0,0157 & 0,2248 & 0,1854 & 0,0524 \\
\hline & & & & & \\
\hline \multicolumn{1}{c}{ Observed Parameter } & D5 & D4 & D3 & D2 & D1 \\
\hline Average Energy & 0,004 & 0,009 & 0,0000 & 0,0000 & 0,000 \\
Normalizing Average Energy & 0,0029 & 0,0004 & 0,0000 & 0,0000 & 0,000 \\
\hline
\end{tabular}

The decomposition energy of these ECG signals is obtained by detail components (D1, D2, D3, D4, D5, D6, D7, D8, D9 and D10) and approximation (A10). Each detail in each decomposition is the extraction of characteristic of ECG .

Table 3. Average Normalization Energy for ECG Signal in a Normal condition.

\begin{tabular}{llrrrrrr}
\hline Observed Parameter & A10 & D10 & D9 & D8 & D7 & D6 \\
\hline Average Energy & 0,1864 & 0,0024 & 0,0108 & 0,0166 & 0,0360 & 0,0442 \\
Normalizing Average Energy & 1 & 0,0128 & 0,0128 & 0,0891 & 0,1932 & 0,2369 \\
\hline \multicolumn{2}{c}{ Observed Parameter } & D5 & D4 & D3 & D2 & D1 \\
\hline Average Energy & 0,0024 & 0,0003 & 0,0000 & 0,0000 & 0,000 \\
Normalizing Average Energy & 0,0130 & 0,0014 & 0,0000 & 0,0000 & 0,000 \\
\hline
\end{tabular}

Table 4. Average Normalization Energy for ECG Signal in a Normal condition.

\begin{tabular}{llrrrrc}
\hline \multicolumn{1}{c}{ Observed Parameter } & A10 & D10 & D9 & D8 & D7 & D6 \\
\hline Average Energy & 0,0196 & 0,0002 & 0,0002 & 0,0006 & 0,0111 & 0,0034 \\
Normalizing Average Energy & 1 & 0,0118 & 0,0084 & 0,0282 & 0,5668 & 0,1711 \\
\hline
\end{tabular}

\begin{tabular}{lccccr}
\hline \multicolumn{1}{c}{ Observed Parameter } & D5 & D4 & D3 & D2 & D1 \\
\hline Average Energy & 0,0021 & 0,0001 & 0,0000 & 0,0000 & 0,000 \\
Normalizing Average Energy & 0,1045 & 0,0068 & 0,0000 & 0,0001 & 0,000 \\
\hline
\end{tabular}




\section{Conclusion}

Here's the conclusion of the research:

1. The heart signal data used in this study is still analog image, so that it is necessary to pre-processing the data processing of ECG signal. The pre-procecessing phase includes scanning, changing images to grayscale, median filters, determining threshold values and morphology.

2. Emotional research through ECG signals was conducted by previous researchers. In this research, the characteristic extraction uses wavelet transform. It can be concluded that the symlet type in wavelet transformation method is suitable and can be used to decompose the ECG signal to level 10 in a happy, normal and sad condition. The disadvantage of this method is we have to decompose multiple signals repeatedly to get the average energy value and normalized average energy from the observed emotions.

3. The average energy value of decomposition normalization can be seen in humans in a happy, normal and sad condition. The average energy value in happy condition at level 8 is 0.2021 . The normal condition at level 6 is 0.0442 . The sad condition at level 7 is 0.111 .

4. Based on the comparison of this research with studies similar to the title "Analysis of emotional conditions based on electrocardiogram signals" and "Image extraction of ECG signals on human emotions using wavelet transformation", we can concluded that both researches have different ways. Overall, the ICA feature extraction method is better than wavelet transformation methods.

\section{References}

[1] R. D. Wulansari, "Sistem Pemantauan Kesehatan Manusia Berbasis Jaringan Sensor Nirkabel," pp. $1-6$.

[2] D. B. Geselowitz, "On the theory of the electrocardiogram," Proc. IEEE, vol. vol 77, no. 6, pp. 857$876,1989$.

[3] B. Sumanto, "Ekstraksi dan Klasifikasi Isyarat EKG Berbasis Transformasi Wavelet dan Jaringan Neural Backpropagation,” Universitas Gadjah Mada, 2012.

[4] R. M.Rangayyan, "Biomedical Signal Analysis (A Case-Study Approach)," Wiley-IEEE Press, p. $509,2002$.

[5] M. Akay, ““'Wavelet Application in Medicine",” Biomed. Eng. Conf, vol. 5, pp. 50-56, 1997.

[6] D. N. K. Hardani, O. Wahyunggoro, H. A. Nugroho, and N. Faisal, "Analysis of emotional condition based on electrocardiogram signals," Proc. 2014 Int. Conf. Electr. Eng. Comput. Sci. ICEECS 2014, no. November, pp. 152-157, 2014.

[7] M. W. Gifari, H. Zakaria, and R. Mengko, "Design of ECG Homecare:12-lead ECG acquisition using single channel ECG device developed on AD8232 analog front end," Proc. - 5th Int. Conf. Electr. Eng. Informatics Bridg. Knowl. between Acad. Ind. Community, ICEEI 2015, pp. 371-376, 2015.

[8] U. Satija, B. Ramkumar, and M. S. Manikandan, "Automated ECG noise detection and classification system for unsupervised healthcare monitoring," IEEE J. Biomed. Heal. Informatics, vol. 22, no. 3, pp. 722-732, 2018.

[9] J. Proulx, R. Clifford, S. Sorensen, D. Lee, and J. Archibald, "Development and Evaluation of a Bluetooth EKG Monitoring Sensor."

[10] K. Stefan, "Heartbents Hint at Personality Traints." .

[11] J. Kim and E. André, "Emotion recognition based on physiological changes in music listening.," IEEE Trans. Pattern Anal. Mach. Intell., vol. 30, no. 12, pp. 2067-83, Dec. 2008. 
[12] B. S. Naresh, "Simulation of phisiological signals using wavelets," 2007.

[13] A. Rizal, "Wireless LAN Electrocardiograph (ECG)," pp. 15-20, 2010.

[14] F. Agra, S. Member, D. Hatzinakos, and A. K. Anderson, "ECG Pattern Analysis for Emotion Detection," pp. 1-14, 2011.

[15] F. Wang, C., \& Wang, "An emotional analysis method based on heart rate variability.," in In Biomedical and Health Informatics (BHI), 2012, pp. 104-107.

[16] J. Cai, G. Liu, and M. Hao, "The Research on Emotion Recognition from ECG Signal," 2009 Int. Conf. Inf. Technol. Comput. Sci., vol. 9, pp. 497-500, Jul. 2009.

[17] P. Busono, “Algoritma Untuk Deteksi QRS Sinyal EKG,” Pros. semiloka Teknol. Simulasi dan Komputasi Serta Apl., pp. 1-7, 2004.

[18] L. J. A. M, "A brief review: history to understand fundamentals of electrocardiography," J. Community Hosp. Intern. Med. Perspect, vol. 2, p. 14383, 2012.

[19] P. Pengoperasian, "Petunjuk Pengoperasian EKG Bionet 2000," 2000.

[20] W. Abu-Ain, S. N. H. S. Abdullah, B. Bataineh, T. Abu-Ain, and K. Omar, "Skeletonization Algorithm for Binary Images," Procedia Technol., vol. 11, no. Iceei, pp. 704-709, 2013. 